\title{
Interpolation of recurrence and hashing entanglement distillation protocols
}

\author{
Karl Gerd H. Vollbrecht and Frank Verstraete \\ Max-Planck-Institut für Quantenoptik, Hans-Kopfermann-Str. 1, D-85748 Garching, Germany
}

(Dated: October 10, 2018)

\begin{abstract}
We construct new entanglement distillation protocols by interpolating between the recurrence and hashing protocols. This leads to asymptotic two-way distillation protocols, resulting in an improvement of the distillation rate for all mixed Bell diagonal entangled states, even for the ones with very high fidelity. We also present a method how entanglement-assisted distillation protocols can be converted into non-entanglement-assisted protocols with the same yield.
\end{abstract}

\section{INTRODUCTION}

One of the big achievements in entanglement theory has been to prove that it is possible to get around the effects of decoherence using an amount of resources that only scales linearly with the number of pure resources one would need. Entanglement distillation and quantum error correction enables to distribute maximally entangled states between different parties in the present of a noisy environment and imperfect quantum channels. This basic task is one of the requirements, if we want to apply the new applications provided by Quantum information theory in a realistic unperfect situation.

Entangled states, in particular pure maximally entangled states, are used as a resource in many protocols in quantum information theory and entanglement of distillation is the most reasonable measure for entanglement respecting this resource character. Given $n$ copies of a bipartite state $\rho$, where the both subsystems are respectively owned by two distinct physicists called Alice and $B o b$, the task is to transform these states into $m$ copies of maximally entangled states. To fulfill this task, Alice and Bob are allowed to make any sequence of local operations on her/his site and they can coordinate their efforts via a classical channel. The set of operations accessible in this way is called LOCC (local operations and classical communication). The best possible rate $m / n$ they could archive (in the asymptotic limit of many copies) defines the distillable entanglement. The latter quantifies in some sense the amount of useful entanglement contained in the state $\rho$. Furthermore, one can think of a situation, where Alice and Bob have already a (maybe infinite) pool of predistilled maximally entangled states. The goal is now to enlarge the number of maximally entangled states in their pool at the end of the protocol, but in between maximally entangled states can be abused for establishing non local operation. Such a protocol is called an entanglement assisted protocol.

Generically, starting with a finite number $n$ of copies, it is never possible to generate maximally entangled states by means of LOCC operations. Purely maximally entangled states can only be achieved by an asymptotic protocol, i.e., in the limit of the number of copies going to infinity.

The construction of such asymptotic distillation protocols leading to a non zero rate is a nasty task and es- sentially all known protocols are only improved versions of the hashing/breeding distillation protocol presented in [1, 2], which is adapted to Bell diagonal states of a two qubit system. This protocol was recently generalized to arbitrary states in arbitrary dimensions [3].

The hashing/breeding protocol is a so called one way distillation protocol, what means, that the classical information is only sent in one direction, e.g. from Alice to Bob, but not in the other way. The great advantage of such kind of protocols is, that they are directly related to quantum error correction codes [2]. But it is well known, that one way distillation protocols are not optimal 2].

One easy way to upgrade a one way distillation protocol to a two way distillation protocol, is to add a prefixed two way operation, like a pre-selection of states based on a measurement outcome, acting respectively only on a few copies. The states produced this way are used as new input for the one way distillation protocol. The prototype of such a protocol is the recurrence protocol, where two copies of a states are mapped by a two way operation to a higher entangled states, which is distilled by the (one way) hashing protocol. Much effort has been spent to optimize or modify such kind of recurrence methods 6, $9,11,12$. The problem that all this protocols share, is that the two way communication part is not asymptotic, resulting in an improvement of the distillation rates only for states with relatively low entanglement.

The present paper is devoted to develop the first asymptotical two way distillation protocol. The following results are obtained:

- We provide an entanglement assisted two way distillation protocol for Bell diagonal two qubit states.

- We show this protocol to give a strictly positive improvement for all Bell diagonal states, expect for the low rank cases, where the hashing protocol was already known to be optimal.

- We show how to further improve the rates and calculate some examples on Werner states.

- We show how to transform this protocols into distillation protocols, that did not need any pre-distilled entanglement.

- We hope to give a better understanding of the breeding protocol and its relation to the recurrence protocol. 


\section{PRELIMINARIES}

The new distillation protocol we want to introduce will be applied to Bell diagonal states on a two qubit system. In particular we will discuss the case of Werner states. Let us start with a short introduction to these kinds of states and briefly recall same basics about the fundamental operations used in the hashing/breeding protocol.

For a two qubit Hilbert space $\mathcal{H}=\mathbb{C}^{2} \otimes \mathbb{C}^{2}$ there exists a basis of maximally entangled states given by the so called four Bell states:

$$
\begin{aligned}
\psi_{00} & =\frac{1}{\sqrt{2}}(|00\rangle+|11\rangle) \\
\psi_{01} & =\frac{1}{\sqrt{2}}(|00\rangle-|11\rangle) \\
\psi_{10} & =\frac{1}{\sqrt{2}}(|10\rangle+|01\rangle) \\
\psi_{11} & =\frac{1}{\sqrt{2}}(|10\rangle-|01\rangle) .
\end{aligned}
$$

The projectors onto these Bell states will be denoted by $P_{i j}=\left|\psi_{i j}\right\rangle\left\langle\psi_{i j}\right|$. We will in the following consider states of the form

$$
\rho_{\lambda}=\sum_{l, k=0}^{1} \lambda_{k l} P_{k l},
$$

which are called Bell diagonal states. These states are paramerized by the four eigenvalues $\lambda:=$ $\left\{\lambda_{00}, \lambda_{01}, \lambda_{10}, \lambda_{11}\right\}$. Bell diagonal states play an important role in entanglement theory [1, 2, 10], especially in distillation theory. Every entangled two qubit state can be transformed to an entangled Bell diagonal states by means of LOCC operations [21]. So the understanding of distillation protocols for Bell diagonal states gives a deep insight in the distillation of arbitrary states.

A Bell diagonal states is separable, if and only if none of its eigenvalues exceeds $\frac{1}{2}$. It is well known, that every entangled Bell diagonal state can be distilled by a combination of the recurrence and the breeding/hashing protocol 2], but the distillation rates archived so far, seem to be far away from an optimum and are much to low to be used as a good lower bound for distillable entanglement.

A Bell diagonal state is called a Werner state 4, 5], if all its eigenvalues $\lambda_{i j}$ except one, e.g. $\lambda_{00}$ are equal. Such a state can be written as

$$
\rho_{f}=f P_{00}+\frac{1-f}{3}\left(\mathbf{1}-P_{00}\right)
$$

The free parameter $f \equiv \lambda_{00}$ is called fidelity. A Werner state is entangled, if and only if $f>0.5$.

As in the case of the known hashing, breeding or recurrence protocol, our protocol will completely stay in the framework of Bell diagonal states, i.e. it will only consist of operations, that map many copies of Bell states to many copies of Bell states. In detail, we will use only bilateral CNOT operations and local measurements.

An essential ingredient for any distillation protocol acting on two qubit systems is the CNOT operation. The CNOT operation is defined by

$$
C|i, j\rangle=|i,(i+j)\rangle,
$$

where the first tensor factor is called the source and the second the target. The addition $(i+j)$ should be read as modulo 2. It is readily verified that a bilateral CNOT operation (BCS), where both parties in a bipartite system apply CNOT operations locally on a tensor product of two Bell states, acts as

$$
(C \otimes C)\left|\psi_{i j}\right\rangle \otimes\left|\psi_{k l}\right\rangle=\left|\psi_{i, j+l}\right\rangle \otimes\left|\psi_{k+i, l}\right\rangle,
$$

here the first tensor product on the l.h.s. in (7) corresponds to the Alice|Bob split, whereas the others correspond to the source|target split. A bilateral CNOT operation maps two copies of a Bell state again to two copies of a Bell state.

In the framework of Bell states, i.e. maximally entangled states, every outcome of a local measurement on Alice's or Bobs side is completely random. Therefore, local measurement makes only sense, if Alice and Bob compare their result. If Alice and Bob make both a measurement in the $|0\rangle,|1\rangle$ basis, the difference of their outcomes will tell them whether it was one of the Bell states $\psi_{00}, \psi_{01}$ or one of the states $\psi_{10}, \psi_{11}$. So Alice and Bob can "measure" together the " $i$ " of an unknown Bell state $\psi_{i j}$. In the same way they can measure " $j "$ using the $|0+1\rangle,|0-1\rangle$ basis. We will refer to such local measurements as local Bell measurements.

\section{KNOWN PROTOCOLS}

Our new protocol is based one the known hashing/breeding protocol and the recurrence protocol. To explain the new protocol it is extremely helpful to give a briefly sketch of these to methods. Later on we will explain our new results. Anybody feeling quite familiar with these protocols can immediately move to section IV

\section{A. The recurrence method}

The recurrence method takes as input two copies of a Bell diagonal state $\rho_{\lambda}$, one called the target state, the other the source state. The idea is to apply a bilateral CNOT operation on two copies of the state $\rho_{\lambda}$ and then making a local Bell measurement on the target state. The source states is kept whenever the measurement outcomes of Alice and Bob coincide, otherwise it is discarded. The overlap of the resulting state with the maximally entangled states increases, if the original overlap was larger than $1 / 2$, which can always be obtained for 
entangled Bell diagonal states. The protocol was originally introduced only for Werner states, where at the end of the protocol the resulting state is mapped to a Werner state again. By iterating this method one can produce from a entangled Werner state (Bell diagonal states) a state that is arbitrarily close to a maximally entangled state.

Note that the recurrence method alone does not lead to a non-zero rate since in every round we destroy or discard at least half of the resources (all the target states) and maximally entangled states are only obtained in the limit of infinitely many rounds. To come to a rate, the resulting states after a finite number of recurrence steps are distilled by the hashing/breeding protocol.

\section{B. The breeding protocol}

The breeding protocol is an entanglement assisted distillation protocol adapted to Bell diagonal sates. In addition to the state $\rho_{\lambda}$ Alice and Bob share arbitrarily many maximally entangled states, which they can use during the distillation process. At the end of the protocol they have to give back the maximally entangled states they abused during the protocol.

Assume that Alice and Bob share $n$ copies of a Bell diagonal state $\rho_{\lambda}$

$$
\rho_{\lambda}^{\otimes n}=\sum_{k_{1} \ldots k_{n}, l_{1} \ldots l_{n}} \lambda_{k_{1} l_{1}} \cdots \lambda_{k_{n} l_{n}} P_{k_{1} l_{1}} \otimes \cdots \otimes P_{k_{n} l_{n}} .
$$

An appropriate interpretation of Eq. (8) is to say that Alice and Bob share the state

$$
P_{k_{1} l_{1}} \otimes \cdots \otimes P_{k_{n} l_{n}}:=P_{\vec{S}}
$$

with probability $\lambda_{k_{1} l_{1}} \cdots \lambda_{k_{n} l_{n}}$. Such a sequence of Bellstates can be identified with the string

$$
\vec{S}=\left(k_{1}, l_{1}, \ldots, k_{n}, l_{n}\right)
$$

Note that if Alice and Bob knew the sequence $\vec{S}$, they could apply appropriate local unitary operations in order to obtain the standard maximally entangled state $P_{00}^{\otimes n}$ and thus gain $n$ ebits of entanglement.

It was shown in [1, 2] that given such a string of Bell states and one extra maximally entangled state $P_{00}$ one can check an arbitrarily parity of the string $\vec{S}$, without changing or disturbing the sequence of Bell states. This is done by applying a sequence of bilateral CNOT operations with the extra maximally entangled state acting as target states, and at a time one state of $\vec{S}$ as source state. The sequence of Bell states is unchanged, but the maximally entangled state $P_{00}$ changed to $P_{x 0}$, where $x$ can be any parity check of the vector $\vec{S}$, i.a. x can be chosen to be

$$
x=\sum_{i} S_{i} M_{i}=\langle\vec{S} \mid \vec{M}\rangle
$$

where $\vec{M}$ is an arbitrarily vector with vector components $\in 0,1$. Note that Eq. (11) has to be read modulo 2 . By a local Bell measurement of the target state Alice and Bob can gain the information $x$, destroying the extra entangled state. For more details see [2]. To such a single operation we will refer as parity check $\vec{M}$. Remember that each such parity check cost Alice and Bob one ebit, i.a. one copy from their pool of maximally entangled states.

The main idea of the breeding protocol is to repeat such parity checks until the full sequence $\vec{S}$ is known. If Alice and Bob did need $m$ parity checks to identify a sequence of $2 n$ bits (n qubits), then they gain $n-m$ maximally entangled states, giving them a distillation rate per copy of $1-m / n$.

This identifying process of $\vec{S}$ is done in the limit of the numbers of copies $n$ going to infinity. This gives the advantages to restrict in the identifying process to so called typically codewords $\vec{S}[14]$. Note that at this stage the whole distillation process is translated into a classical problem: Given an classical bit string $\vec{S}$ generated by a probability distribution $\left(\lambda_{i j}\right)$, how many parity checks are necessary to identify the bit string ? The String $\vec{S}$ generated by many copies of the state $\rho_{\lambda}$ contains $n S\left(\rho_{\lambda}\right)$ bits of classical information, where

$$
S\left(\rho_{\lambda}\right)=-\sum_{i j} \lambda_{i j} \log \lambda_{i j}
$$

is the von Neumann Entropy of the state $\rho_{\lambda}$. With each parity check we gain one bit of this information [7]. Per qubit we therefore need $S(\rho)$ ebit of entanglement to identify it, leading to the well known hashing/breeding rate

$$
D_{\text {Hashqubit }}\left(\rho_{\lambda}\right)=1-S\left(\rho_{\lambda}\right)=1-S(\lambda) .
$$

The hashing/breeding rate has shown to be optimal for rank deficient Bell states, i.e., Bell diagonal states of rank two 8.

The same kind of protocol can be adapted to states that are diagonal in a tensor product basis of Bell states, i.e. for states that are diagonal in a basis of the form

$$
\psi_{\overrightarrow{i j}}=\psi_{i_{1} j_{1}} \otimes \psi_{i_{2} j_{2}} \ldots \psi_{i_{m} j_{m}},
$$

i.e. states of the form $\sigma_{\lambda}=\sum_{\overrightarrow{i j}} \lambda_{\overrightarrow{i j}} P_{\overrightarrow{i j}}$. In this case one copy of a state generates not a two bit (one qubit) string, but a $2 m$ bit (n qubit) string. Taking $n$ copies of the state we get a random $2 \mathrm{~nm}$ bit string generated by the probability distribution $\left(\lambda_{\overrightarrow{i j}}\right)$. We can try to identify this string by doing parity checks in the same way we did before. The amount of parity checks per copy is in the same spirit given by $S\left(\sigma_{\lambda}\right)$, but if we identified one copy of the state we now gain $m$ ebits instead of 1 ebit. The distillation rate for these states is therefore given by

$$
D_{\text {Hash }}\left(\sigma_{\lambda}\right)=m-S(\lambda) \text {. }
$$


For more details see [19]. A special type of states that are diagonal in such a basis are many copies of Bell diagonal states, e.g. $\sigma_{\lambda}=\rho_{\lambda}^{\otimes n}$. But since the hashing/breeding rate (15) is additive we gain or lose nothing by applying a breeding protocol to several copies of a Bell state.

\section{THE NEW PROTOCOL}

The new protocol is essentially an asymptotic version of a recurrence step followed by the breeding protocol. The new idea is to take many copies of the state $\rho$ and start by distilling states which have the 'same parity'.

\section{A. Distill parity states}

In the breeding protocol the goal is to get the full information " $i j$ " for every state $\psi_{i j}$. One can also set the goal to get only a part of this information, e.g. we want only to know the $i$ or the $j$ or the parity $i+j$. This correspond to the the information we would get, if we made the corresponding parity check $\langle\{i, j\} \mid \vec{m}\rangle$ on one copy, e.g. $\vec{m}=10,01,11$, which would cost one ebit per copy.

But this information can be obtained in a much cheaper way using exactly the same asymptotic technics as in the breeding protocol. We can write the state as

$$
\rho_{\lambda}=\mu_{0} \rho_{0}+\mu_{1} \rho_{1}
$$

where

$$
\begin{aligned}
\mu_{k} & =\sum_{\langle\{i, j\} \mid \vec{m}\rangle=k} \lambda_{i j} \\
\rho_{k} & =\frac{1}{\mu_{k}} \sum_{\langle\{i, j\} \mid \vec{m}\rangle=k} \lambda_{i j} P_{i j} .
\end{aligned}
$$

If we take now $n$ copies of the state $\rho_{\lambda}$ we get

$$
\rho_{\lambda}^{\otimes n}=\sum_{k_{1} \ldots k_{n}} \mu_{k_{1}} \cdots \mu_{k_{n}} \rho_{k_{1}} \otimes \cdots \otimes \rho_{k_{n}} .
$$

Similarly to (8) an appropriate interpretation of Eq. (19) is to say that Alice and Bob share the state

$$
\rho_{k_{1}} \otimes \cdots \otimes \rho_{k_{n}}
$$

which can be identified with a bit string

$$
\vec{S}^{\prime}=\left(k_{1}, \ldots, k_{n}\right)
$$

To get the information about $\vec{S}^{\prime}$ in the same way we get it for the string $\vec{S}$, we need to make arbitrary parity checks $\vec{M}^{\prime}$ on the vector $\vec{S}^{\prime}$. Fortunately, $\vec{S}^{\prime}$ and $\vec{S}$ described exactly the same sequence of Bell state, the only difference is that $\vec{S}^{\prime}$ contains not the full information. Thus we can translate any parity check $\vec{M}^{\prime}$ on $\vec{S}^{\prime}$ to a parity check $\vec{M}$ on $\vec{S}$ by the simple rule

$$
M_{2 i-1}=m_{1}, M_{2 i}=m_{2}
$$

whenever $M_{i}^{\prime}=1$ and

$$
M_{2 i-1}=0, M_{2 i}=0
$$

whenever $M_{i}^{\prime}=0$. It is readily checked that we can make this way every parity check $\vec{M}^{\prime}$ on $\vec{S}^{\prime}$ paying on ebit of entanglement. To identify $\vec{S}^{\prime}$ in the limit of many copies we therefore need $S\left(\mu_{0}, \mu_{1}\right)$ ebit of entanglement.

So given a state $\rho$, we can decompose the state into $\rho_{0}$ and $\rho_{1}$ with probability $\mu_{0}, \mu_{1}$ by paying $S\left(\mu_{0}, \mu_{1}\right)$ ebit per copy. Doing such a step in our protocol we will refer as an asymptotic parity check $\vec{m}$. In a completely analog way we define asymptotic parity checks for states that are diagonal in the basis (14). The parity vector $\vec{m}$ is in this case a $2 m$ bit string. The rule to translate parity checks is given by

$$
M_{(m-1) i+1, \ldots, m i}=\vec{m}
$$

whenever $M_{i}^{\prime}=1$ and

$$
M_{(m-1) i+1, \ldots, m i}=\overrightarrow{0}
$$

whenever $M_{i}^{\prime}=0 . \quad \overrightarrow{0}$ denotes a vector of length $m$ containing only zeros.

If Alice and Bob make enough asymptotic parity checks, they gain the full information about every copy, making this procedure equivalent to a distillation. Indeed, the original breeding protocol consist of two of such asymptotic parity checks. First a '10' asymptotic parity check and afterwards a '01' asymptotic parity check. Since for the entropy holds

$$
\begin{aligned}
S(\lambda) & =\left(\lambda_{0}+\ldots+\lambda_{m}\right) S\left(\left[\lambda_{0}, \ldots, \lambda_{m}\right]\right) \\
& +\left(\lambda_{m+1}+\ldots+\lambda_{n}\right) S\left(\left[\lambda_{m+1}, \ldots, \lambda_{n}\right]\right) \\
& +S\left(\lambda_{1}+\ldots+\lambda_{m}, \lambda_{m+1}+\ldots+\lambda_{n}\right),
\end{aligned}
$$

we gain or lose nothing by doing the distillation in several asymptotic parity checking steps. Here $S\left(\left[p_{1}, \ldots, p_{n}\right]\right)$ denotes the entropy of the normalized probability distribution, i.e. $S\left(\left[p_{1}, \ldots, p_{n}\right]\right):=S\left(p_{1} / N, \ldots, p_{n} / N\right)$ with $N=\sum_{i} p_{i}$.

\section{B. The improved protocols}

The key of our new distillation protocols is to distill several copies of a Bell diagonal state $\sigma_{\lambda}=\rho_{\lambda}^{\otimes m}$, by a sequence of asymptotic parity checks. After each such step, we get two kind of states, the one that pass the asymptotic parity check (parity equal zero) and the one that fail the test (parity equal one). Instead of continuing the distillation by testing further parities, we consider two new possibilities, 
- We can decide to drop some of the states, dependent on the outcome of an asymptotic parity check. Indeed, this improves our distillation rate, iff the state we drop has a negative breeding rate, i.e. $m-S(\rho)<0$.

- We make a local Bell measurement on one of the $m$ two qubit systems. Dependent on the measurement output we get a new $m-1$ two qubit system state. Before doing the measurement, we also allow to apply Alice and Bob local unitaries. But we restrict to such unitaries, that map many copies of Bell states again to many copies of Bell states.

\section{Asymptotic recurrence}

First we present a protocol, that is very similar to making one recurrence step and afterwards distilling the state with the breeding protocol. The main difference is, that the recurrence step is somehow made in an asymptotic way. We take two copies of a Bell diagonal $\rho_{\lambda}$. Then we asymptotically check the parity 1010 of these two copies. The state passes this test, if it is in one of the states

$$
\text { 0000, 0001, 0100, 0101, 1010, 1011, 1110, 1111, }
$$

it fails the test if it is in one of the states

$$
\text { 0010, 0011, 0110, 0111, 1000, 1001, 1100, } 1101 .
$$

Therefore, $\rho_{\lambda}^{\otimes 2}$ passes the parity check with probability $p_{\text {even }}=\left(\lambda_{00}+\lambda_{01}\right)^{2}+\left(\lambda_{10}+\lambda_{11}\right)^{2}$ and is afterwards in the state

$$
\rho_{\text {even }}=\frac{1}{p_{\text {even }}} \sum_{i j l} \lambda_{i j} \lambda_{i l} P_{i j} \otimes P_{i l} .
$$

With probability $p_{\text {odd }}=2\left(\lambda_{00}+\lambda_{01}\right)\left(\lambda_{10}+\lambda_{11}\right)$ it fails and is is in the state

$$
\rho_{\text {odd }}=\frac{1}{p_{\text {odd }}} \sum_{i j l} \lambda_{i j} \lambda_{(i+1) l} P_{i j} \otimes P_{(i+1) l}
$$

This step costs $S\left(p_{\text {odd }}, p_{\text {even }}\right)$ ebit per copy of $\rho_{\lambda}^{\otimes 2}$. Similarly to the recurrence protocol, we decide to drop one of these two possible outcomes. A calculation for Werner states shows, that $\rho_{\text {odd }}$ has a negative breeding rate for every entangled Werner states $\rho_{f}$. So we decide to drop all odd states and continue to distill the even states. The distillation rate is then given by

$$
-S\left(p_{\text {odd }}, p_{\text {even }}\right)+p_{\text {even }}\left(2-S\left(\rho_{\text {even }}\right)\right) .
$$

$S\left(p_{\text {odd }}, p_{\text {even }}\right)$ is what we have to pay for the first parity check. Afterwards we continue with probability $p_{\text {even }}$ to distill $\rho_{\text {even }}$. Normalized to one copy of the state $\rho_{\lambda}$ we obtain

$$
-S\left(p_{\text {odd }}, p_{\text {even }}\right) / 2+p_{\text {even }}\left(1-S\left(\rho_{\text {even }}\right) / 2\right),
$$

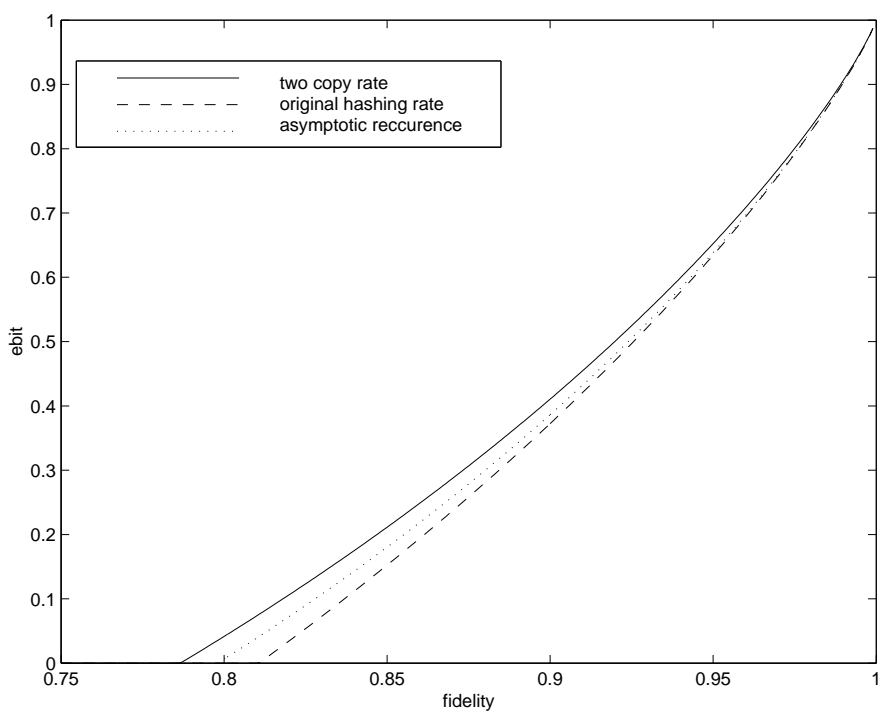

FIG. 1: Distillation rates for Werner states.

which is plotted in Fig 1 for Werner states. Surprisingly, we get an improvement to the known breeding protocol over the full range of the fidelity and not only for low fidelities, like it is known for combined hashing recurrence protocols.

\section{2. the 2-copy rate}

Although the breeding rate of the state $\rho_{\text {odd }}$ is negative, the state may be still entangled and even distillable. So one can improve the distillation rate, by applying an alternative distillation protocol onto $\rho_{\text {odd }}$. One way to get a positive rate out of $\rho_{\text {odd }}$ is to make a local Bell measurement on one of the two qubit systems. If the measurement outcomes of Alice and Bob do not coincide (correspond to the states 10 or 11), they knew that the remaining state has to be 00 or 01 , because they knew, the overall state had an odd parity in the first bit of each two qubit system. If the measurement outcomes coincide (00 or 01), they knew the remaining state in one of the states 10,11 . So for both outcomes they end up in a rank two Bell diagonal state, for which they knew, that the breeding protocol gives them the optimal distillation rate. In fact, the mixture of 00,01 is always equally weighted and therefore separable, so they can drop it. But the mixture of 10,11 can give positive contribution to the overall distillation rate. The distillation rate obtained with this protocol for Werner states is plotted in Fig [

The distillation rate for arbitrary Bell diagonal two qubit states using this protocol is easily calculated to be

$$
\begin{aligned}
D_{2 c}(\vec{\lambda})= & 1-S(\rho) \\
& +\frac{p_{\text {odd }}}{4}\left[S\left(\left[\lambda_{00}, \lambda_{01}\right]\right)+S\left(\left[\lambda_{11}, \lambda_{10}\right]\right)\right]
\end{aligned}
$$

So it is a real improvement to the known hashing/breeding rate, since we beat this rate by the extra 


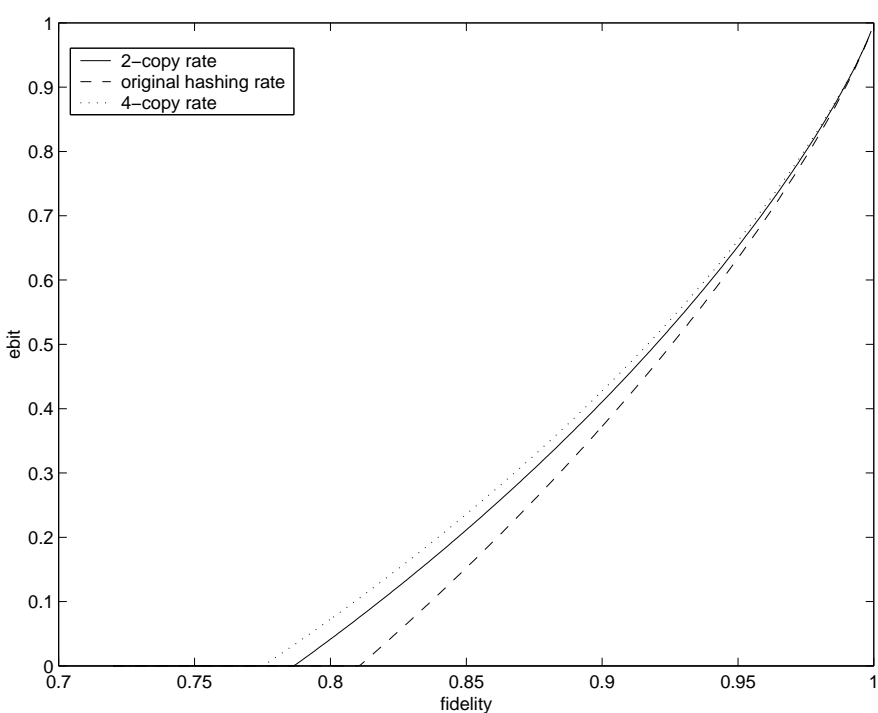

FIG. 2: Distillation rates for Werner states.

term (34), which is always larger or equal to zero. Indeed, (34) is zero if and only if the state has rank less than two. We have a strictly positive improvement for all Bell diagonal states, with the only exception for those cases, where the hashing/breeding rate was known to be optimal.

\section{Further optimization}

What rates can we achieve using protocols consisting only out of asymptotic parity checks and local Bell measurements?

We can start from more than two copies of the state and construct in the same spirit protocols consisting out of asymptotic parity checks and local Bell measurements. The result of one such protocol starting with four copies $\rho_{\lambda}^{\otimes 4}$ is plotted in Fig 2 for Werner states. Using selfrecurrent programming, it is in principle possible to prove optimality of such schemes within the class of operations described above. Note however that the computational cost for doing this optimization is superexponential in the number of copies involved in the protocol, and proving optimality therefore does not seem to be feasible for more than 6 copies. It is however clear that taking more copies can only increase the distillation rate. Unfortunately, we did not manage to find an easy way to construct reasonable good protocols for more and more copies; in the asymptotic limit, this would maybe even lead to an exact expression for the entanglement of distillation.

On Werner states the above 2-copy protocol is essentially the best possible protocol using asymptotic hashing steps and local measurements. We have done an optimization for Werner states over all protocols, which include arbitrary asymptotic hashing steps, local Bell measurements and arbitrary local unitary operations, map-

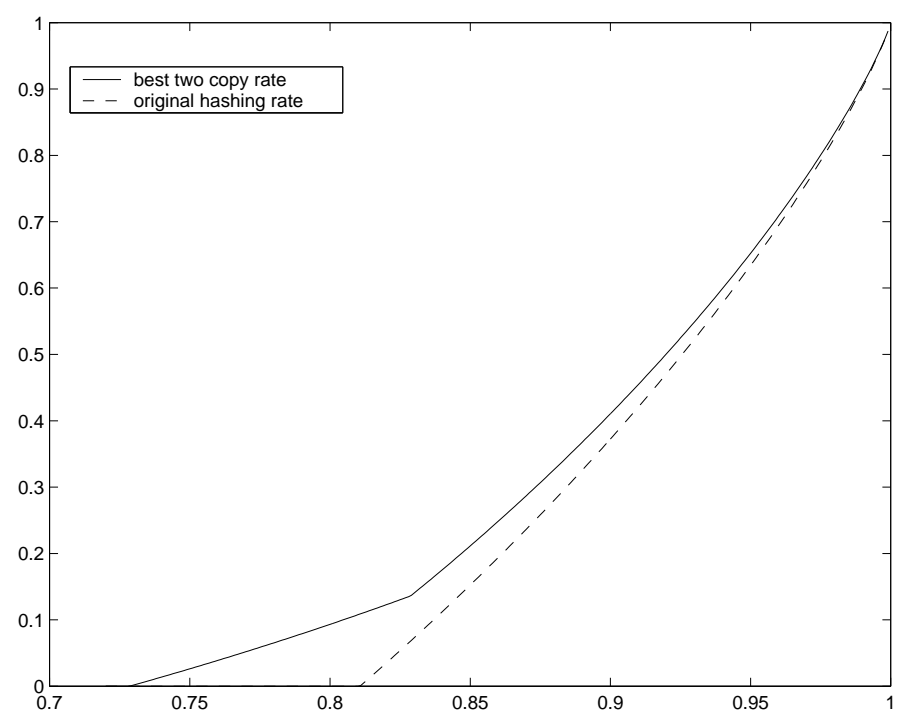

FIG. 3: Distillation rates for Werner states.

ping multi-copies of Bell states into multi-copies Bell states. The number of such protocols is finite. The Asymptotic hashing steps can be parameterized by the $2 n$ bit string $\vec{m}$. All local unitaries that map multi-copies of Bell states to multi-copies of Bell states have been characterized in [6]. For two copies of Bell states there exists up to local equivalence 120 different possibilities. The number of possible asymptotic parity checks or local Bell measurements that are possible, until the state is known or completely destroyed is also finite.

So one has to test for a given Bell diagonal state a finite (but very large) set of possible protocols. The result of this optimization for all protocols starting from two copies of a Werner state is plotted in Fig [3 For high fidelities the above 2-copy protocol gave the best results. For low fidelities the standard recurrence followed by the optimized hashing, which is included in this kind of protocols, is optimal.

For more than two copies of the state we were not able to do the full optimization over all possible protocols, because the number of protocols is too large. By making a good guess, we could find protocols up to 5 copies giving further small improvements. But so far, we are unable to give a systematic way of producing $n$-copy-protocols.

\section{NON ENTANGLEMENT ASSISTED PROTOCOLS}

We will now show, how the above protocols can be transformed into protocols that do not need any predistilled maximally entangled states. The idea is to start with an non entanglement assisted protocol to get a starting pool of maximally entangled states for the entanglement assisted protocol. To distill this starting pool, we will only use a sub-linear amount of copies, so that in the asymptotic limit, we will not affect the obtained rates. 
In detail, we start with $k^{-1} \sqrt{n}+n$ copies of the state $\rho$, where $k>0$ is the distillation rate obtained by any non entanglement assisted protocol, e.g. we can use several recurrence steps followed by the hashing protocol. We use this non entanglement assisted activating protocol to distill $\sqrt{n}$ maximally entangled states out of the first $k^{-1} \sqrt{n}$ copies. At this step let us assume, that the activating protocol does this without any error, i.e. we get perfect maximally entangled states.

We use these $\sqrt{n}$ maximally states as resource for our entanglement assisted protocol to distill $(1+r) \sqrt{n}$ copies of the state $\rho$ gaining $(1+r) \sqrt{n}$ new maximally entangled states. At this step we also assume the entanglement assisted protocol to work perfect for a finite amount of copies. Here $r$ denotes the rate obtained by the entanglement assisted protocol. With these maximally entangled states we distill the next block of $(1+r)^{2} \sqrt{n}$ copies from the $n$ copies of $\rho$ and so on for all the rest of it. The obtained rate for this non entanglement assisted protocol will be $r$, independent of the rate $k$ of the activating protocol at the beginning, because the extra $k^{-1} \sqrt{n}$ copies of $\rho$ will be vanish compared to $n$ for $n$ going to infinity.

Of course, in contradiction to our assumption, none of the protocols will work perfect for any finite number of copies and the errors, that will occur, may accumulate and destroy the whole protocol. So to claim this above protocol to be a valid distillation protocol, we have to ensure that the success probability of the protocol goes to one if $n$ goes to infinity.

So we now want to bound the success probability of the protocol described above. First of all we only can guarantee our protocol work, if the starting $\sqrt{n}$ maximally entangled states were correct, i.e. if the activating protocol succeeded. Let us call this probability $p(n)$, with $p(n)$ going to zero, if $\mathrm{n}$ goes to infinity. Second we call $q(n)$ the probability, that the entanglement assisted protocol works well. Then the probability that the above protocol works can be bounded by

$$
p_{\text {succ }} \geq p(\sqrt{n}) q(\sqrt{n})^{\sqrt{n}} .
$$

In the worst case we have to repeat the entanglement as- sisted protocol $\sqrt{n}$ times successfully with only $\sqrt{n}$ copies of the state $\rho$ as input. Indeed, this probability can go to zero, if $q(n)$ scales in a wrong way. The probability $p(\sqrt{n})$ is no problem at all, as long as it goes to one for large $n$.

So we only have to ensure, that $q\left(n^{\prime}\right)^{n^{\prime}}$ with $n^{\prime}=\sqrt{n}$ goes to one as $n^{\prime}$ goes to infinity. What we therefore need is an approximation of the scaling of the success probability $q(n)$ for our new protocols. The entanglement assisted protocol described above will succeed, if the sequences correspond to so called typical sequences. The probability of being a typical sequence goes exponentially to one [20], so we can bound for $n^{\prime}$ large enough $q\left(n^{\prime}\right)>\left(1-k e^{-c n^{\prime}}\right)$. So the overall success probability can be bounded by

$$
p_{\text {succ }} \geq p(\sqrt{n})\left(1-k e^{-c \sqrt{n}}\right)^{\sqrt{n}},
$$

which goes to one, if $n$ goes to infinity.

\section{CONCLUSION}

We have presented the first distillation protocol, where two way communication is included in an asymptotical sense. The distillation rates attained this way beat the known hashing/breeding rate over the whole range of entangled Bell diagonal states. Since the hashing/breeding protocol is the last step for all recurrence like distillation schemes, we can improve all these distillation protocols. Furthermore we proved how any entanglementassisted distillation protocol can be converted into a nonentanglement-assisted distillation protocol with the same rate. An important open problem is to find a systematic way for constructing n-copy distillation protocols; this could ultimately lead to an exact expression of the entanglement of distillation.

Work supported by EU IST projects, the DFG, and the Kompetenznetzwerk Quanteninformationsverarbeitung der Bayerischen Staatsregierung.
[1] C.H. Bennett, G. Brassard, S. Popescu, B. Schumacher, J. Smolin, W.K. Wootters, Phys. Rev. Lett 76, 722 (1996).

[2] C.H. Bennett, D.P. DiVincenzo, J.A. Smolin, W.K. Wootters, Phys. Rev. A 54, 3824 (1996).

[3] I. Devetak and A. Winter, quant-ph/0304196

[4] R.F Werner, Phys. Rev. A 40 (1989) 4277-4281.

[5] This states are also known as isotropic states. On a two qubit system they differ from the original Werner states only by a local transformation, whereas in higher dimensions isotropic and Werner states are substantially different.

[6] J.Dehaene, M. Van den Nest, B. De Moor and F. Verstraete, Phys. Rev. A 67, 022310 (2003).
[7] Indeed, to show that we gain 1 bit of information and not less is the tricky part in the proof. See [2].

[8] E. Rains, quant-ph/9707002 (1997).

[9] D. Deutsch, A. Ekert, R. Jozsa, C. Macchiavello, S. Popescu, and A. Sanpera, Phys. Rev. Lett. 77, 2818 (1996).

[10] M. Horodecki and P. Horodecki, Phys. Rev. A 59, 4206 (1999).

[11] G. Alber, A. Delgado, N. Gisin, I. Jex, quant-ph/0102035 (2001).

[12] E.N. Maneva and J.A. Smolin, quant-ph/0003099 (2000).

[13] E. Rains, quant-ph/0008047 (2000).

[14] B. Schumacher, Phys. Rev. A 51, 2738 (1995).

[15] K.G.H. Vollbrecht and R.F. Werner, Phys. Rev. A 64, 
062307 (2001).

[16] A. Kent et al., Phys.Rev.Lett. 83, 2656 (1999); F. Verstraete et al., Phys. Rev. A 64, 010101(R) (2001).

[17] F. Verstraete and H. Verschelde, Phys. Rev. Lett. 90, 097901 (2003)

[18] A. Ambainis and D. Gottesman, quant-ph/0310097

[19] K.G.H. Vollbrecht and M.M. Wolf, Phys. Rev. A 67,
012303 (2003).

[20] T.M. Cover and J.A. Thomas, Elements of Information Theory, Wiley Series in Telecommunication.

[21] An optimal single-copy procedure to do this with probability 1 has been found in 17], while the optimal probabilistic protocol is given in [16]. 